\title{
Disambiguation of Source and Trajectory Non-Stationarities of a Moving Acoustic Source
}

\author{
Sai Gunaranjan Pelluri and T. V. Sreenivas \\ Department of Electrical Communication Engineering, Indian Institute of Science, Bangalore, India-560012 \\ Email: saigunaranjanpelluri@gmail.com, tvsree@ece.iisc.ernet.in
}

\begin{abstract}
It is well known that when there is a relative motion between the transmitter (source) and receiver, a Doppler shift is observed in the spectral content of the received signal. In this paper, we investigate a scenario where the source signal itself has an innate spectral non-stationarity in addition to the non-stationarity introduced by the source motion relative to the receiver. Using only a single microphone recording, we show that these two kinds of non-stationarities are distinguishable and propose a method of separating them. Towards this, we propose a novel scheme of simulating the signal from a source traversing an arbitrary trajectory. The proposed simulation mechanism employs band-limited interpolation and nonuniform sampling to incorporate an acoustic source generating an arbitrary band-limited signal and moving along an arbitrary trajectory.
\end{abstract}

\section{INTRODUCTION}

Moving objects and acoustic sources are an ubiquitous presence around us. They include animals, birds, vehicles and more recently even robots! These sources generate signals in the form of electro-magnetic radiations, acoustic wave patterns, etc. The signals emitted by these sources may be used to classify one type of source from another but they do not reveal much about the source motion per se. However, when these sources emit signals during motion, the recorded signals exhibit some interesting properties which can help reveal the motion dynamics of the source. This is particularly of interest in the context of acoustic scene analysis (ASA) by either man or machine (robot). With the advances in robotics, human-machine interaction has thrown open new challenges. Hu-bots or humanoid robots are being developed to interact with their environment and also help humans. Equipped with a wide range of sensors comprising of microphones, cameras, proximity sensors, etc, the hubots are attempting to mimic intelligent and purposeful behaviour. In this regard, tasks such as identifying distress calls, localizing a certain source, communicating with other moving robots (swarm of robots), etc. are being widely addressed. What makes it even more interesting and challenging is that since the hubots need to make decisions while in motion, the characteristics of the acoustic signals do get altered due to the relative motion between a source and a sensor.

From the well known Doppler effect, we know that when there is relative motion between a receiver and a source generating a single frequency, the perceived frequency at the receiver undergoes a shift. The Doppler equation for a moving source and a stationary receiver can thus be expressed as[1]:

$$
f_{R}=f_{T}\left(1+\frac{v_{r}}{c}\right)
$$

where $f_{T}$ and $f_{R}$ are the frequencies of the transmitted and received waves respectively and $c$ is the speed of the wave in the homogenous acoustic medium. $v_{r}$ is the velocity of the source in the direction of the stationary receiver and it is positive if the source is moving towards the receiver and is negative when the source is moving away from the receiver. In general, $v_{r}$ can also be time-varying and the above equation can be expressed as

$$
f_{R}=f_{T}\left(1+\frac{v_{r}(t)}{c}\right) .
$$

We see that even though the source transmits a stationary tone, the received signal is a time-varying tone due to the motion dynamics of the source reflected in $v_{r}(t)$. Based on the nature of the relative motion, the Doppler shift can in general be time-varying. This results in frequency modulations in the received signal and its analysis requires signal processing through joint spectro-temporal methods. Now, if the source transmits a non-stationary signal (e.g. amplitude modulated and frequency modulated), further nonstationarity is introduced due to the motion dynamics of the source with respect to the receiver. In this work, we quantify these two kinds of spectral non-stationarities using only a single microphone recording for the analysis.

Since the Doppler effect captures the motion dynamics between the source and receiver, analysing signals generated by moving sources can aid in characterising the source and its motion dynamics. This finds applications in various domains such as aircraft localization [2], simulation of realistic battlefield effects for troop training [3], virtual reality creation, etc. Some of the popular examples of moving acoustic source signals include bird chirps, horn sound of fast moving vehicles, etc. We explore some of the interesting characterestics of signals resulted by moving acoustic sources.

\section{DISAMBIGUATION OF SOURCE NON-STATIONARITY (SNS) AND TRAJECTORY NON-STATIONARITY (TNS)}

Let us consider an acoustic source generating nonstationary (AM-FM) signals. In the above discussion, we 


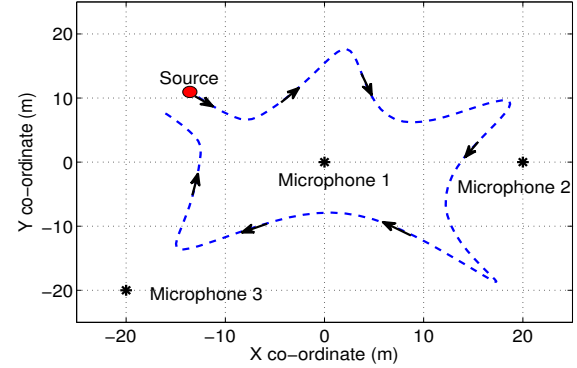

Fig. 1. Illustration of an arbitrary trajectory traversed by a moving source and a 3 microphone receiver configuration.

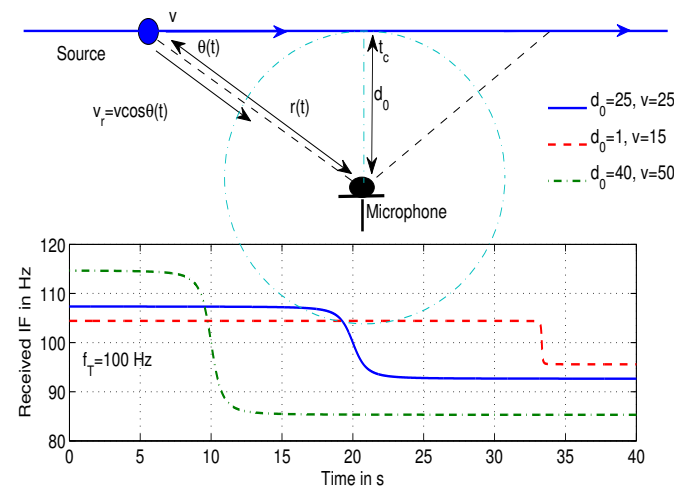

Fig. 2. (Color online) Schematic of a linear path, constant velocity moving source and a stationary receiver. Also shown is a plot of the IF trajectories for different combinations of $d_{0}(\mathrm{~m})$ and $v(\mathrm{~m} / \mathrm{s})$.

have seen how non-stationarity is introduced in the received signal due to the motion dynamics of a tonal source. Let us refer to this as trajectory non-stationarity (TNS). We now show that for sources generating AM-FM signals with innate non-stationarity, which we refer to as source non-stationarity (SNS), additional non-stationarity gets introduced in the received signal due to the motion trajectory of the source. We also show that these two kinds of non-stationarities namely source non-stationarity (SNS) and trajectory nonstationarity (TNS) can be decoupled in the received signal.

Let $s(t)$ denote the transmitted signal which is both amplitude-modulated and frequency-modulated (AM-FM) and is generated by a moving acoustic source and let $x(t)$ be the corresponding signal at the receiver. So, we have

$$
s(t)=a(t) \sin \phi(t),
$$

and

$$
x(t)=b(t) \sin \phi(t-\tau(t)),
$$

where the instantaneous delay is

$$
\tau(t)=\frac{r(t)}{c} .
$$

$r(t)$ denotes the radial distance between the source and the microphone and $c$ is the velocity of sound in the medium. To avoid notational clutter, we replace $\tau(t)$ with $\tau$ noting that $\tau$ is also a function of time. Thus, IF of the received signal is given by:

$$
\omega(t)=\frac{d}{d t} \phi(t-\tau)=\phi^{\prime}(t-\tau)\left(1+\frac{v_{r}(t)}{c}\right),
$$

where $v_{r}(t)$ denotes the radial velocity of the source in the direction of the receiver and is given by:

$$
v_{r}(t)=-\frac{d}{d t} r(t)
$$

To get an insight into $\omega(t)$, let us use the Taylor series expansion of $\phi(t-\tau(t))$, upto third order terms. Thus, we get:

$$
\phi(t-\tau)=\phi(t)-\tau \phi^{\prime}(t)+\frac{\tau^{2}}{2 !} \phi^{\prime \prime}(t)-\ldots
$$

Taking derivative of Eqn. (7) on both sides with respect to $t$ we obtain:

$$
\begin{array}{r}
\phi^{\prime}(t-\tau)\left(1+\frac{v_{r}(t)}{c}\right)=\phi^{\prime}(t)-\left[\tau \phi^{\prime \prime}(t)-\phi^{\prime}(t) \frac{v_{r}(t)}{c}\right] \\
+\frac{1}{2}\left[\tau^{2} \phi^{\prime \prime \prime}(t)-2 \phi^{\prime \prime}(t) \tau \frac{v_{r}(t)}{c}\right]+\ldots
\end{array}
$$

Neglecting the third and higher order terms and substituting the above expression in Eqn. (5) and rearranging we get:

$$
\begin{aligned}
\omega(t) & =\left[1+\frac{v_{r}(t)}{c}\right]\left[\phi^{\prime}(t)-\phi^{\prime \prime}(t) \frac{r(t)}{c}\right] \\
& =\left[1+\frac{v_{r}(t)}{c}\right] \phi^{\prime}(t)\left[1-\tau(t) \frac{\phi^{\prime \prime}(t)}{\phi^{\prime}(t)}\right]
\end{aligned}
$$

For higher order non-stationary signals such as quadratic or cubic chirps, the term $\frac{\phi^{\prime \prime}(t)}{\phi^{\prime}(t)}$ tends to zero and hence the above expression can be simplified to:

$$
\omega(t) \approx \underbrace{\left[1+\frac{v_{r}(t)}{c}\right]}_{T N S} \underbrace{\left[\phi^{\prime}(t)\right]}_{S N S} .
$$

From the above expression, it is evident that the nonstationarity in the received signal can be decomposed into two components scaling each other. The first component is due to the trajectory of the source, denoted as trajectory non-stationarity (TNS). Note that TNS is a dimensionless quantity and hence has no units. The second component contains the non-stationarity due to the innate nature of the transmitted signal (i.e $\phi(t)$ ) and we refer to this as the source non-stationarity (SNS). Thus, to a certain approximation, we can see that received IF is simply the source IF $\left(\phi^{\prime}(t)\right)$ scaled by the TNS. This goes to indicate that in principle, we can undo the doppler effect (de-dopplerization) by choosing a source signal whose IF varies as

$$
\phi^{\prime}(t)=\frac{1}{\left[1+\frac{v_{r}(t)}{c}\right]} .
$$


We illustrate this through an example simulation, justifying the analysis. Let us consider a source moving along a spiral trajectory as shown in Fig. 9. The TNS $\left(1+\frac{v_{r}(t)}{c}\right)$ for a spiral path is shown in Fig. 3(a). We now consider a source generating a signal whose IF varies according to Eqn. (11) (Fig. 3(b)). The source is then simulated to move along the spiral path. The de-dopplerized IF of the received signal $x(t)$ is shown in Fig. 3(c). A nearly constant $\omega(t)$ is obtained. Now, applying logarithm on both sides of Eqn. (10) we obtain

$$
\log \omega(t) \approx \log [T N S]+\log [S N S]
$$

Thus, in the log domain, the two non-stationarities appear as a linear combination. Since there are two parts to the non-stationarity of the received signal, we can have two situations. Either the rate of variation of the SNS component can be greater than the TNS component or vice-versa. We shall consider both the cases through simulation examples.

\section{A. $S N S>T N S$}

A transmitted signal is simulated with a Gaussian envelope (AM) centered at 0.5 seconds and FM given by

$$
f_{T}(t)=f_{c}+f_{m}(t),
$$

where $f_{c}=100 \mathrm{~Hz}$ is the carrier, and $f_{m}(t)=5 \sin (30 \pi t)$ is the modulation around the carrier. The source is moving along a linear trajectory with a velocity of $60 \mathrm{~m} / \mathrm{s}$ and a perpendicular distance of $5 \mathrm{~m}$ from the receiver (Fig. 2). The sampling frequency is chosen as $1 \mathrm{kHz}$. The received signal IF is estimated using the extrema-sample (ES) [4], [8] based IF estimation technique. Based on the above analysis, the received signal's IF $\left(f_{R}(t)\right)$ is given by

$$
\begin{aligned}
f_{R}(t) & =f_{T}(t)\left(1+\frac{v_{r}(t)}{c}\right) \\
& =\left(f_{c}+f_{m}(t)\right)\left(1+\frac{v_{r}(t)}{c}\right) \\
& =\hat{f}_{c}(t)+\hat{f_{m}}(t) .
\end{aligned}
$$

From the above equation, we see that the received IF is composed of two additive components and so an empirical mode decomposition (EMD) [9] can be used to separate out the individual components namely $\hat{f}_{c}(t)$ and $\hat{f_{m}}(t)$. For the considered example, the TNS can be analyzed as a relatively slower variation compared to the fast-varying FM of the source signal (SNS). Hence, $f_{m}(t)$ is not influenced much by the source motion as compared to $f_{c}$ and so $\hat{f_{m}}(t) \approx f_{m}(t)$. Fig. 4 shows the received signal IF and its additive components for the considered example. Note that in Fig. 4(b), the TNS component is coupled with the carrier and hence it has the units of $\mathrm{Hz}$. The estimated IF of the doppler induced carrier frequency $\hat{f}_{c}(t)$ can be used for the estimation of source motion parameters ([5]) since it contains information about source motion dynamics while the IF estimate of $f_{m}(t)$ can be used to analyze acoustic features of the source signal.

\section{B. $S N S<T N S$}

We simulate a moving source whose transmitted frequency increases quadratically as a function of time, i.e.

$$
f_{T}(t)=100+2 t^{2}
$$

The source is made to oscillate continuously along a circular path with a linear velocity of $20 \mathrm{~m} / \mathrm{s}$ and a microphone positioned at $(-8,0)$ as shown in Fig. 7 . In this case, $f_{T}(t)$ (SNS) can be viewed as a slower variation as compared to the motion of the source (TNS). Fig. 5(a) shows IF of the received signal. Clearly, the IF consists of two components namely, a slowly varying quadratic chirp (SNS) and a rapidly oscillating component due to the oscillatory motion of the source (TNS). The individual non-stationarities are obtained as follows. First, a logarithm operation is applied on the IF of the received signal to obtain $\log \omega(t)$. Then, the EMD is performed on $\log \omega(t)$ to obtain the individual components namely $\log [T N S]$ and $\log [S N S]$. The actual nonstationarities are then obtained by taking antilog on each of $\log [T N S], \log [S N S]$. An interesting observation from Fig. 5 (a) is that the dynamic range of the oscillations increases as the underlying IF of quadratic chirp increases. This is a direct implication of the two non-stationarities occurring as a product as evident from Eqn. (10).

\section{SIMULATION OF ACOUSTIC SOURCE TRAVERSING ARBITRARY TRAJECTORY}

The simulation of moving acoustic sources finds its applications in fields such as music and virtual reality. There exists broadly three approaches for simulating a moving source: i) perceptual approach ii) physical approach and iii) filtering techniques. The perceptual approaches [6] make use of specific perceptual cues (such as sound intensity, timbre) for the synthesis of realistic moving sounds. The physical approaches involve modelling sound fields using models based on sound wave propagation equations. Some of the popular techniques include ambisonics, surround sound, etc. The perceptual and physical approaches are high end techniques and are not suitable for the problem at hand. The filtering based techniques involve a sliding window based convolution (overlap-add) of the signal with a temporally and spatially varying room impulse response (RIR) [7]. However, the major drawback with this technique is the computational complexity because it involves computing a convolution in every time frame. Also, the RIR for every point that the source traverses needs to be pre-computed and stored. In this context, we develop a novel scheme of receiver signal generation which is based on the interpolation of essentially band-limited time-varying signal samples, along with nonuniform sampling of the source signal.

The analysis of a tonal source moving along a linear path leads to closed form expressions revealing the interplay of various parameters. However, when we have sources generating non-stationary signals and traversing arbitrary paths, it is not possible to obtain closed form expressions 


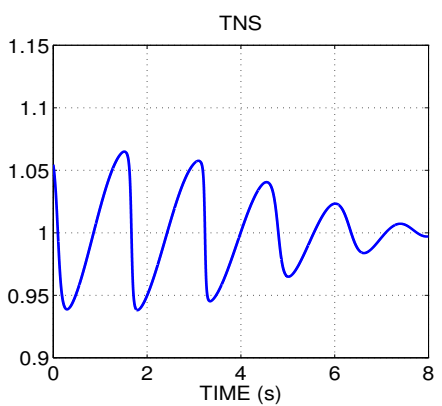

(a)

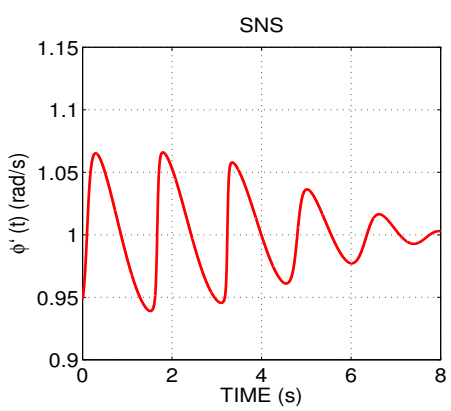

(b)

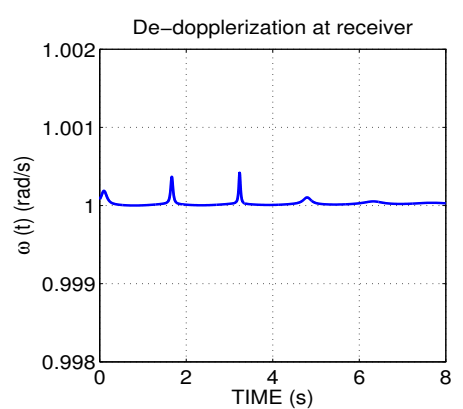

Fig. 3. (Color online) a) TNS of a source moving along a spiral path; b) IF of a source signal chosen to vary as $\phi^{\prime}(t)=1 / T N S$; c) de-dopplerized IF $(\mathrm{rad} / \mathrm{sec})$ of $x(t)$ at receiver.

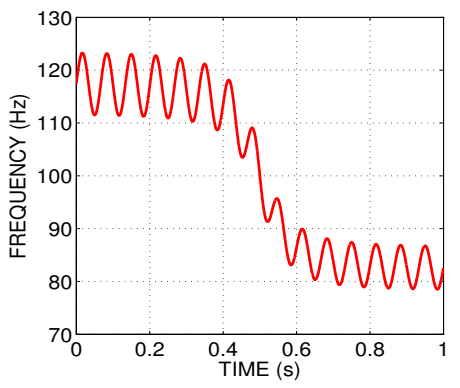

(a)

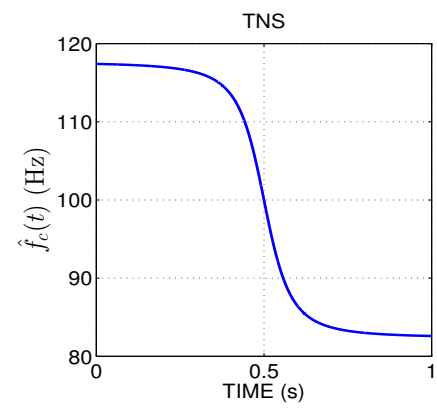

(b)

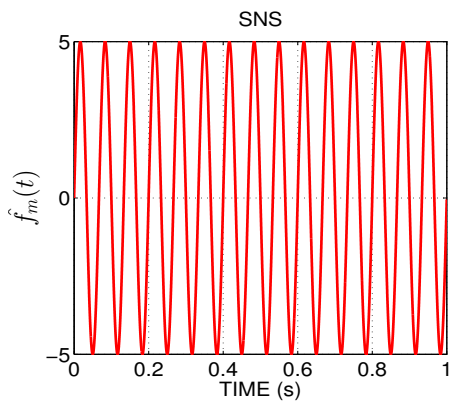

(c)

Fig. 4. (Color online) The received IF and its non-stationary components for an AM-FM transmitted signal. a) IF of the received signal $f_{R}(t)$. b) TNS component induced in the carrier of the transmitted signal $\left(f_{c}\right)$. c) SNS component $\left(f_{m}(t)\right)$ of the received signal.

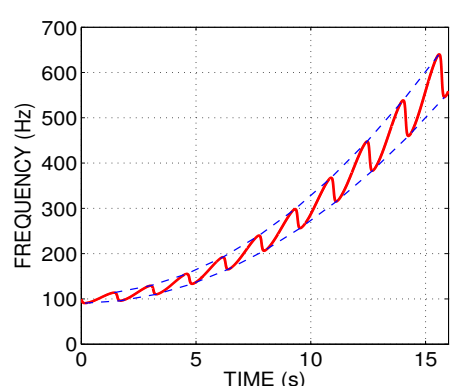

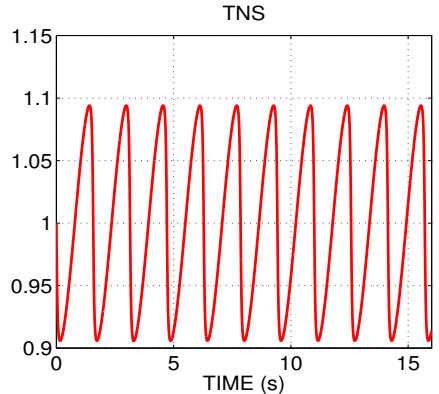

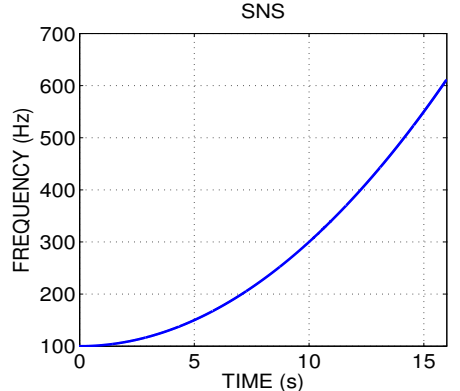

Fig. 5. (Color online) The received IF and its non-stationary components for an AM-FM transmitted signal. a) IF of the received signal in Hz. b) TNS component. c) SNS component of the received signal.

but instead we have to resort to discrete time simulation. Through this, we can analyse the received signal at different microphone locations and then suitably combine the estimated parameters at different locations. The goal of acoustic scene analysis (ASA) being to infer as much information from as few sensors as possible, the analysis through simulation, provides a means of developing new algorithms based on IF for source estimation using multimicrophone deployment.

For an acoustic source traversing along an arbitrary path and generating a signal $s(t)$, the received signal can be modeled as:

$$
x(t)=a(t) s(t-\tau(t)),
$$

where $a(t)=1 / r(t)$ models the time varying amplitude and $\tau(t)=r(t) / c$ models the time-varying delay and $r(t)$ is the instantaneous distance of separation between the source and the receiver. In general, the received signal being nonstationary, has a larger bandwidth (theoretically infinite) even when the transmitted signal is band-limited. Hence, we need to use an over-sampled discrete-time signal model at the receiver. Now, let us consider the received signal $x(t)=x\left[n T_{s}\right]$ that is highly oversampled to cater to the 
larger bandwidth of $x(t)$ compared to the transmitted signal $s(t)$. Thus,

$$
x\left(n T_{s}\right)=\frac{1}{r\left(n T_{s}\right)} s\left(n T_{s}-\tau(n T s)\right) .
$$

Now, let $y\left(n T_{s}\right) \triangleq s\left(n T_{s}-\tau\left(n T_{s}\right)\right)$. So we have the discrete samples of the received signal as

$$
x\left(n T_{s}\right)=\frac{1}{r\left(n T_{s}\right)} y\left(n T_{s}\right) .
$$

Here, $\tau(t)=r(t) / c$ is a continuous function due to the continuous trajectory, causing perturbation on the time samples of $s\left(n T_{s}-\tau\left(n T_{s}\right)\right)$; hence we need interpolation of $s\left(n T_{s}\right)$ to determine $y(n)$ and subsequently $x(n)$. However, if we consider simpler parametric source signals with a closed form expression, such as sinusoids, we don't need interpolation and re-sampling; instead we can directly compute the received signal using the closed form expressions. Thus, we generate $x\left(n T_{s}\right)$ through two steps

- step 1. Bandlimited interpolation of $s\left(n T_{s}\right)$.

- step 2. Re-sampling of the interpolated signal.

Since $\tau(t)$ varies depending on the motion dynamics of the source, the re-sampling turns out to be a non-uniform sampling of the transmitted signal $s(t)$. The interpolation formula is given by

$$
s(t)=\sum_{n=-N / 2}^{+N / 2} s\left(n T_{s}\right) h\left(t-n T_{s}\right) ; h(t) \triangleq \operatorname{sinc}\left(t / T_{s}\right),
$$

Once we have the interpolated signal, we need to sample it at discrete locations given by the vector $\boldsymbol{V}=\left[1 T_{s}\right.$ $\left.\tau\left(1 T_{s}\right), 2 T_{s}-\tau\left(2 T_{s}\right), \ldots, N T_{s}-\tau\left(N T_{s}\right)\right]^{T}$. In this manner, we can generate $y\left(n T_{s}\right)$. Finally, the received signal samples are obtained by multiplying $1 / r\left(n T_{s}\right)$ with $y\left(n T_{s}\right)$. So, in order to determine the received signal of a moving acoustic source, the transmitted signal $s(t)$ and the time delay $\tau(t)$ (which captures the trajectory of the source) with respect to each microphone in the receiver configuration are required.

In a typical simulation analysis, an arbitrary trajectory is chosen by placing multiple points sequentially on an XYZ grid using a GUI. The points are assumed to be sampled from the required source trajectory. A cubic B-spline is used to interpolate the points and obtain smooth functions of $\mathrm{X}, \mathrm{Y}$ and $\mathrm{Z}$ co-ordinates as a function of time. The coordinates $X(t), Y(t)$ and $Z(t)$ are then resampled at points corresponding to $n T_{s}$ of the microphone signal sampling. Thus, the trajectory is obtained as the set of ordered tuples $\left\{X\left(n T_{s}\right), Y\left(n T_{s}\right), Z\left(n T_{s}\right) ; n=0,1,2, \ldots.\right\}$. With the set of ordered tuples defining the trajectory of the acoustic source, the time delay $\tau_{i, j}(n)$ between the $i^{t h}$ source and the $j^{\text {th }}$ microphone is calculated as

$$
\tau_{i, j}(n)=\frac{r_{i, j}(n)}{c}=\frac{\left\|\boldsymbol{P}_{\boldsymbol{i}}(\boldsymbol{n})-\boldsymbol{M}_{\boldsymbol{j}}\right\|_{2}}{c},
$$

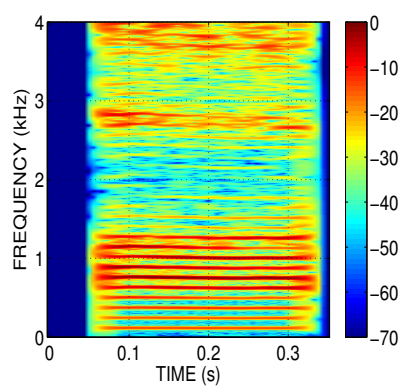

(a)

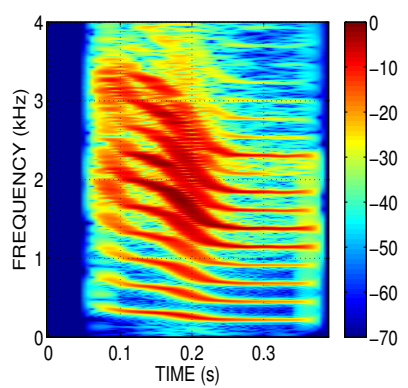

(b)
Fig. 6. (Color online) a) Spectrogram of the stationary vowel ' $/ a /$ '; b) A source generating the stationary vowel ' $/ a /$ ' is simulated to move along a linear path with a constant velocity of $60 \mathrm{~m} / \mathrm{s}$.

where $P_{i}(n)$ is the position vector of the $i^{t h}$ source at $t=$ $n T_{s}$, given by

$$
P_{i}(n)=[X(n), Y(n), Z(n)]^{T},
$$

and $M_{j}$ is the position of the $j^{\text {th }}$ microphone. Once $\tau_{i, j}(n)$ is computed $\forall n$ defined by the trajectory of the source, $x\left(n T_{s}\right)$ is obtained by sampling the interpolated signal $s(t)$ at discrete instants dictated by the sequence $\boldsymbol{V}$ and then multiplying with envelope $1 / r\left(n T_{s}\right)$. Thus, we can consider any arbitrary band limited signal $s(t)$ and obtain the received signal $x(t)$ through the interpolated signal re-sampling. Note that $x[n]$ denotes the samples of the received signal and $X[n]$ denotes the sequence of $X$ co-ordinates of the moving source. Similarly, $y[n]$ denotes the samples of the received signal but without the amplitude modulation $(1 / \mathrm{r}[\mathrm{n}])$ and $Y[n]$ denotes the sequence of $\mathrm{Y}$ co-ordinates of the moving source.

Fig. 6 (a) shows the spectrogram of the stationary vowel sound ' $/ a /$ ' recording. In Fig. 6 (b), the source generating the vowel is simulated to move along a linear path with a constant velocity of $60 \mathrm{~m} / \mathrm{s}$ as explained above.

\section{MOVING SOURCE EXAMPLES}

Let us consider some simple examples of sources generating a stationary tone of $1000 \mathrm{~Hz}$ and moving with a linear velocity of $20 \mathrm{~m} / \mathrm{s}$ along the trajectories shown in part (a) of Figs. 7 to 10. The microphone(s) are considered omnidirectional. Part (b) of each figure shows the corresponding instantaneous frequency (IF) of the received signal. We can observe the periodicity of IF in Figs. 7 and 8. This periodicity corresponds to the periodic motion of the source along the trajectories where as the average IF of $1000 \mathrm{~Hz}$ is the source frequency. Any change in the linear velocity along the periodic path will appear as aperiodicity in the IF. In Fig. 8(b), the IF at the two microphone positions is different for the same source trajectory, yet the IF contours for both the mics share the same periodicity. In Fig. 9, we consider a circular but spiralling in trajectory, resulting in a "damped" IF. Conversely, if the source were spiralling out, the IF would be a growing pattern, clearly distinguishing between a homing-in or homing-out source. Also, the IF is not strictly 

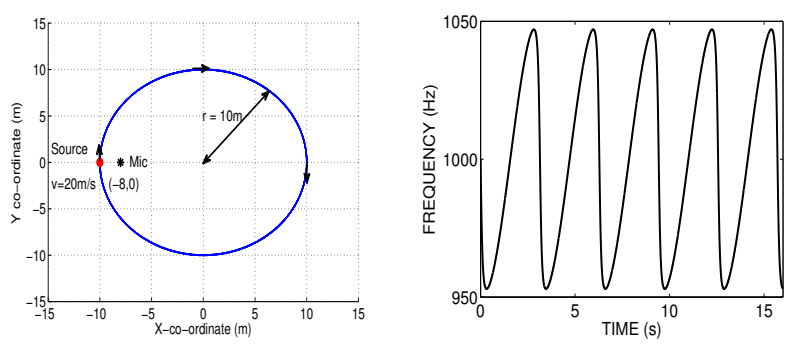

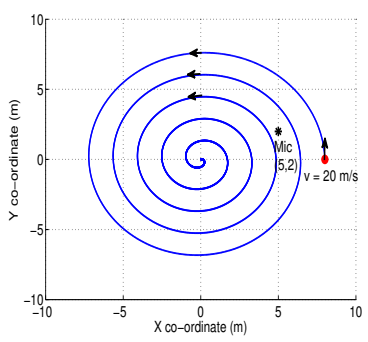

(a)

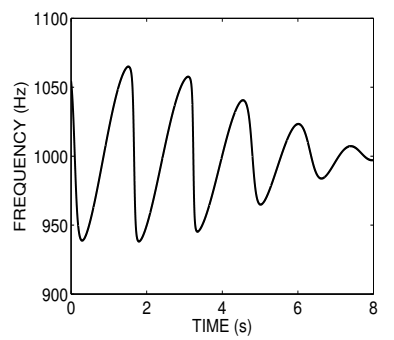

(b)

Fig. 7. a) Source traverses a circular trajectory with microphone fixed at $(-8,0)$; b) IF of signal captured at the microphone.

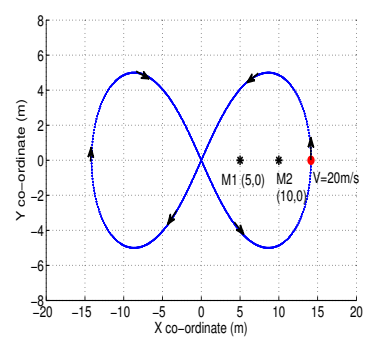

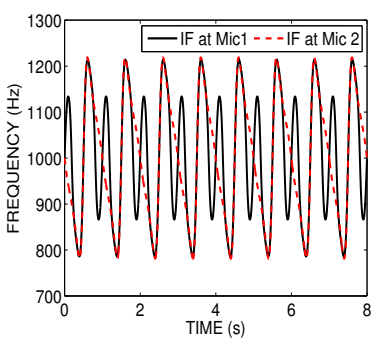

(b)

Fig. 8. (Color online) Source (indicated by red dot) moves in an ' $\infty$ ' shaped trajectory. Microphones (M1, M2) are positioned $5 \mathrm{~m}$ apart from each other as shown.

periodic, rather it is a skewed sinusoid with a decay. We can see that the rate of decay in dynamic range of the oscillating IF is equal to the rate of decay in the radius of the spiral trajectory as the source is homing in. In all the Figures 7 - 9, the microphones are fixed and do not move. In Fig. 10, we consider additional motion of the receiver also. The microphone is made to move in a circular path with its centre at $(6,0)$ and a radius of $2 \mathrm{~m}$. The constant linear velocity of the source is $20 \mathrm{~m} / \mathrm{s}$ while that of the microphone is $10 \mathrm{~m} / \mathrm{s}$. On careful observation, we can see that the IF in this case too is periodic and the periodicity is the least common multiple (LCM) of the source periodic motion and the periodic motion of the receiver.

Through the above interesting simulation examples, we can see that the motion dynamics of the source are well captured in the IF contours of the received signal. Because of the homogenuity of the medium and the superposition principle, any arbitrary source signal comprising several tonal frequencies will also result in corresponding variation of the received IF components.

\section{CONCLUSIONS}

In this paper, we have addressed some interesting aspects associated with signals captured from moving acoustic sources. In particular, we dealt with the spectral nonstationarities that are introduced due to the motion of the source. We have also theoretically quantified the two types of spectral non-stationarities associated with moving acoustic sources and have shown that they can be decoupled under certain conditions. This can aid in understanding the motion

Fig. 9. (Color online) Source (red dot) moves in a spiral path with the microphone fixed at $(5,2)$. The radius of the spiral path decreases linearly at a rate of $1 \mathrm{~m} / \mathrm{s}$ starting with an initial radius of $8 \mathrm{~m}$, resulting in a damped IF pattern.
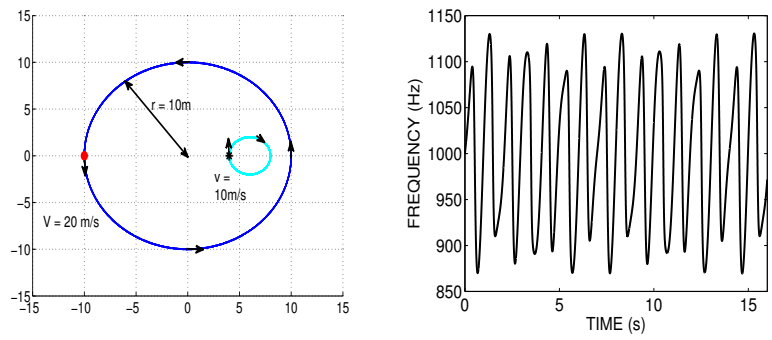

(b)

Fig. 10. (Color online) Both source (red dot) and receiver (*) move in circular paths but with differnt radii and velocities.

dynamics and signal characteristics of the source independently. Through few real life experiments and simulations, we have shown that a joint spectro-temporal analysis is essential to understand the behaviour of fast moving acoustic sources.

\section{REFERENCES}

[1] Allan D Pierce et al., Acoustics: an introduction to its physical principles and applications, vol. 20, McGraw-Hill New York, 1981.

[2] Brian G Ferguson and Barry G Quinn, "Application of the shorttime fourier transform and the wigner-ville distribution to the acoustic localization of aircraft," The Journal of the Acoustical Society of America, vol. 96, no. 2, pp. 821-827, 1994.

[3] Joseph I Statman and Eugene R Rodemich, "Parameter estimation based on doppler frequency shifts," IEEE transactions on aerospace and electronic systems, vol. 23, no. 1, pp. 31-39, 1987.

[4] Sai Gunaranjan Pelluri and Thippur V. Sreenivas, "Arbitrary trajectory estimation of a moving acoustic source," in Audio Engineering Society Convention 138, May 2015.

[5] N. Sharma, S.G. Pelluri, and T.V. Sreenivas, "Moving sound source parameter estimation using a single microphone and signal extrema samples," in IEEE International Conference on Acoustics, Speech and Signal Processing (ICASSP), 2015, April 2015, pp. 2329-2333.

[6] John M Chowning, "The simulation of moving sound sources," Journal of the Audio Engineering Society, vol. 19, no. 1, pp. 2-6, 1971.

[7] Eric A Lehmann and Anders M Johansson, "Diffuse reverberation model for efficient image-source simulation of room impulse responses," IEEE Transactions on Audio, Speech, and Language Processing, vol. 18, no. 6, pp. 1429-1439, 2010.

[8] Neeraj Kumar Sharma and Thippur V. Sreenivas, "Event-triggered sampling using signal extrema for instantaneous amplitude and instantaneous frequency estimation," Signal Processing, vol. 116, no. 0, pp. $43-54,2015$.

[9] Azadeh Moghtaderi, Patrick Flandrin, and Pierre Borgnat, "Trend filtering via empirical mode decompositions," Computational Statistics \& Data Analysis, vol. 58, no. 0, pp. 114 - 126, 2013, The Third Special Issue on Statistical Signal Extraction and Filtering. 